\title{
Distribution of Fos-Like Immunoreactivity in the Medullary Reticular Formation of the Rat after Gustatory Elicited Ingestion and Rejection Behaviors
}

\author{
Lisa A. DiNardo ${ }^{1}$ and Joseph B. Travers ${ }^{1,2}$ \\ Departments of ${ }^{1}$ Psychology and ${ }^{2}$ Oral Biology, The Ohio State University, Columbus, Ohio, 43210
}

The distribution of neurons in the medullary reticular formation (RF) activated by the ingestion of sucrose or rejection of quinine was examined using standard immunohistochemical techniques to detect the expression of the Fos protein product of the immediate-early gene c-fos. Double-labeling techniques were used to gain further insight into the possible functional significance of RF neurons exhibiting Fos-like immunoreactivity (FLI). Compared with sucrose and unstimulated controls, quinine elicited significantly more FLI neurons in three specific RF subdivisions: parvocellular reticular nucleus (PCRt), intermediate reticular nucleus (IRt), and dorsal medullary reticular nucleus (MdD). Moreover, the number of FLI neurons in the RF of quinine-stimulated animals was significantly correlated with the degree of oromotor activity. Thus, the distinct distribution of FLI neurons throughout the RF after quinine may reflect the acti- vation of a specific oral rejection circuit. The double-labeling results indicated a high degree of segregation between FLI neurons and premotor projection neurons to the hypoglossal nucleus (mXII) retrogradely labeled with Fluorogold. Thus, although there were a significant number of double-labeled neurons in the RF, the major concentration of premotor projection neurons to $\mathrm{mXII}$ in IRt were medial to the preponderance of FLI neurons in the PCRt. In contrast, there was substantial overlap between FLI neurons in the RF and labeled fibers after injections of the anterograde tracer, biotinylated dextran into the rostral (gustatory) portion of the nucleus of the solitary tract. These results support a medial (premotor)/lateral (sensory) functional topography of the medullary RF.

Key words: c-fos; medullary reticular formation; brainstem; ingestion; rejection; rat
One of the fundamental roles of gustation is to discriminate palatable from unpalatable, often toxic substances. In the rat (Woods, 1964; Grill and Norgren, 1978b) as well as other species (Steiner, 1973; Berntson and Micco, 1976), the underlying circuitry for this discrimination is located in the caudal brainstem, because decerebrate animals respond appropriately with stereotyped ingestion and rejection behaviors after gustatory stimulation. Although the location of the "switch" from ingestion to rejection is unknown, a role for the medullary reticular formation (RF) is suggested. Both the first-order central gustatory relay [the rostral nucleus of the solitary tract (NST)] and the second order gustatory relay [the parabrachial nucleus $(\mathrm{PBN})$ ] project to specific regions of the RF (Norgren, 1978; Travers, 1988; Beckman and Whitehead, 1991; Becker, 1992; Shammah-Lagnado et al., 1992; Halsell et al., 1996; Karimnamazi et al., 1996; reviewed by Travers, 1993), which in turn provide the main input to the oromotor nuclei (Holstege et al., 1977; Travers and Norgren, 1983; Vertes et al., 1986; Ter Horst et al., 1991). Thus, the RF is implicated as a polysynaptic substrate through which gustatory information influences oromotor behavior.

In recent years, the expression of the Fos protein product of the immediate-early gene c-fos has been used as a functional marker for mapping polysynaptic neuronal activity in the central nervous

Received Dec. 19, 1996; revised Feb. 13, 1997; accepted Feb. 21, 1997.

This work, a chapter of a dissertation (L.A.D.), was supported by National Institutes of Health Grant R01 DC00417 to J.B.T. We thank Drs. Susan P. Travers, Christopher B. Halsell, and Mark Dinkins for their helpful comments on this manuscript. Kevin J. Urbanek and Hecheng Hu provided excellent technical assistance.

Correspondence should be addressed to Joseph B. Travers, College of Dentistry, The Ohio State University, 305 West 12th Avenue, Columbus, OH 43210.

Copyright (C) 1997 Society for Neuroscience $0270-6474 / 97 / 173826-14 \$ 05.00 / 0$ system after sensory stimulation (e.g., Onoda, 1992; Gieroba and Blessing, 1994; Jasmin et al., 1994b; Carstens et al., 1995; McEchron et al., 1996), often coupled with motor behavior (Barajon et al., 1992; Jasmin et al., 1994a; Miller and Ruggiero, 1994; Wallois et al., 1995). Fos immunohistochemistry has also been used to specifically examine brain regions involved in feeding regulation (e.g., Olson et al., 1993; Rinaman et al., 1993; Fraser et al., 1995; Emond and Weingarten, 1995), gustatory processing (Yamamoto et al., 1994; Harrer and Travers, 1996), and conditioned taste aversion (Swank and Bernstein, 1994; Houpt et al., 1994; Swank et al., 1995). The purpose of the present study was to determine the distribution of RF neurons exhibiting Fos-like immunoreactivity (FLI) associated with gustatory elicited ingestion and rejection behaviors. In the rat, the rejection response consists of large amplitude mouth openings (gapes), accompanied by a complex sequence of lingual movements (Grill and Norgren, 1978a; Travers and Norgren, 1986; DiNardo and Travers, 1994). Because this gape response appears as a resequencing of the motor pattern of the lick response but also shows parallels to the emetic response in other species, the control of rejection may be closely associated with the circuitry involved in the production of ingestive rhythmic behaviors (Moriyama, 1987; Nozaki et al., 1993; reviewed by Travers et al., 1997) or emesis (Borison and Wang, 1949; Mehler, 1983; Fukuda and Koga, 1991). The potential role of RF neurons exhibiting FLI in gustatory oromotor circuitry was determined with double-labeling techniques to examine the extent to which FLI neurons project to the oromotor nuclei (c-fos/retrograde labeling) and whether FLI neurons in the RF overlap with projections from the NST (c-fos/anterograde labeling). 


\section{MATERIALS AND METHODS}

\section{C-fos/retrograde tracing study}

\section{Surgical procedures}

Adult, male Sprague Dawley rats $(n=18)$ weighing between 275 and 425 $\mathrm{gm}$ were used in these experiments. All animals were maintained on a normal $12 \mathrm{hr}$ light/dark cycle. Nine to $14 \mathrm{~d}$ before behavioral testing, rats were anesthetized with sodium pentobarbital (Nembutal, $50 \mathrm{mg} / \mathrm{kg}$, i.p.) with supplemental doses $(0.1 \mathrm{ml})$ administered, when needed, to maintain a surgical level of anesthesia characterized by hindlimb areflexia. Body temperature was monitored and maintained at $37^{\circ} \mathrm{C}$ throughout the surgery. Each rat was fitted with two intraoral cannulae constructed of PE 100 tubing to allow experimenter-controlled infusions of tastants directly into the oral cavity (Grill and Norgren, 1978a). The cannulae were inserted into the oral mucosa, posterior and lateral to the first maxillary molars, and exited through an incision on the dorsal surface of the head.

After cannulae insertion, rats were positioned in a stereotaxic instrument (David Kopf Instruments, Tujunga, CA) equipped with blunt ear bars, and the skull was horizontally leveled. A $2 \mathrm{~mm}$ trephine hole was drilled into the skull $6.8 \mathrm{~mm}$ posterior and $0.15 \mathrm{~mm}$ lateral to $\lambda$, and the dura was reflected to provide access to the hypoglossal nucleus (mXII). The hypoglossal nucleus was located using microstimulation (Travers and Norgren, 1983). The dorsoventral coordinates of mXII from the brain surface were determined at a site at which lingual movements were elicited by passing a low level of current [ $7 \mu \mathrm{A}, 0.1 \mathrm{msec}$ pulses, 250 pulses per second (pps)] through the microelectrode. A glass micropipette with a tip diameter of $25 \mu \mathrm{m}$ was then filled with a $2 \%$ solution of the fluorescent retrograde tracer Fluorogold (FG; Fluorochrome, Inc., Englewood, $\mathrm{CO}$ ) dissolved in $0.1 \mathrm{~m}$ cacodylate buffer or a $4 \%$ solution dissolved in physiological saline. To verify that the pipette was actually placed within mXII, small amounts of current $(20 \mu \mathrm{A}, 0.1 \mathrm{msec}$ pulses, $250 \mathrm{pps}$ ) were passed through the pipette to evoke lingual movements. Once the pipette was situated midway between the dorsal and ventral borders of mXII, approximately $10 \mathrm{nl}$ of FG was pressure injected (20-30 psi, $20-30$ msec pulses) into mXII. In two additional rats, FG was injected into the trigeminal motor nucleus. The pipette was left in place for $5 \mathrm{~min}$ after injection.

After tracer injection, the intraoral cannulae were secured to the skull with dental acrylic applied around the cannulae and skull screws. The incision was closed with surgical clips, and a topical antibiotic (NMC Laboratories, Inc., Glendale, NY) was applied to the wound. All rats were given penicillin (20,000 U, s.c./day) for 3-4 postoperative days. During the recovery period, rats were fed a mixture of powdered rat chow and Crisco to facilitate weight gain. For $7 \mathrm{~d}$ before behavioral testing, rats were placed in a clear Plexiglas observation chamber for $1 \mathrm{hr}$, followed by a $30 \mathrm{~min}$ period of intraoral water stimulation $(50 \mu \mathrm{l}$ every $2 \mathrm{~min})$ at roughly the same time each day. Although animals did not receive as many intraoral fluid deliveries as they would later during testing, this adaptation procedure mimicked the overall testing protocol with the aim of reducing the novelty and stress of the testing paradigm. Testing subsequently took place at the same time of day as the adaptation procedure for a given animal.

\section{Testing procedures}

On the test day, rats were placed into the Plexiglas observation chamber for a $1 \mathrm{hr}$ adaptation period before testing. After the adaptation period, rats were given multiple $50 \mu$ infusions of a particular tastant for 18 trials (eight $50 \mu \mathrm{l}$ infusions per trial delivered every $6-8 \mathrm{sec}$ ) over a $36 \mathrm{~min}$ period to yield a total volume of $7.2 \mathrm{ml}$ of fluid. Intertrial intervals were 2 min. Hedonically distinct tastants were chosen to elicit different behavioral responses. One group of animals $(n=6)$ received $1.0 \mathrm{M}$ sucrose to elicit an ingestive sequence of behaviors, including rhythmic jaw movements, tongue protrusions, and lateral tongue protrusions. A second group of animals $(n=6)$ received $0.003 \mathrm{M}$ quinine monohydrochloride to elicit an aversive behavioral sequence consisting of gapes, chin rubs, forelimb flails, head shakes, rearing, and passive rejection (Grill and Norgren, 1978a). For subsequent analysis of behavior, rats were videotaped during the entire 36 min testing period via a video camera aimed at a mirror positioned beneath the observation chamber. A control group of animals $(n=6)$ underwent the same surgical and adaptation protocols as the experimental groups but were not given any tastants during the 36 min testing period. This unstimulated control group provided an index of "baseline" FLI in awake rats.

\section{Immunohistochemistry}

Forty-five min after the test session, rats were deeply anesthetized with Nembutal $(150 \mathrm{mg} / \mathrm{kg})$ and perfused transcardially with $150-200 \mathrm{ml}$ of warm $\left(37^{\circ} \mathrm{C}\right) 0.1 \mathrm{M}$ PBS, $\mathrm{pH} 7.4$, followed by $200-400 \mathrm{ml}$ of a cold $4 \%$ paraformaldehyde solution, $\mathrm{pH}$ 6.8. In some rats $(n=8), 1.25 \%$ acrolein (Polysciences, Inc., Warrington, PA) was added to the paraformaldehyde solution to produce a more consistent fixation. The brain was removed and placed in $0.1 \mathrm{M}$ phosphate buffer (PB) with $20 \%$ sucrose or, when needed, post-fixed in a $20 \%$ sucrose paraformaldehyde solution overnight. The hindbrain was cut transversely at $40 \mu \mathrm{m}$ on a freezing microtome into three series. One series was immediately mounted onto gelatin-coated slides for subsequent analysis of the FG injection site. A second series was stored in a cryoprotectant solution for further processing, if needed. The tissue from the last series was processed for FLI using a protocol described by Harrer and Travers (1996). Tissue from "positive control" animals was reacted in tandem with tissue from each rat in this study to monitor the quality of the immunohistochemical reactions. Two different positive control manipulations were chosen. One positive control group $(n=2)$ was anesthetized with Nembutal $(50 \mathrm{mg} / \mathrm{kg}$, i.p.), and the chemical irritant capsaicin $(0.001 \mathrm{M})$ was injected ipsilaterally into various regions of the oral cavity (e.g., anterior tongue and cheek), as well as swabbed over the cornea. This procedure was repeated four times over a $30 \mathrm{~min}$ period. Forty-five minutes later, the rats were perfused using the same procedure as described above. A second positive control group $(n=$ 3) was given i.p. injections of the endotoxin lipopolysaccharide (LPS; 40 $\mu \mathrm{g} / 0.2 \mathrm{ml})$ and perfused $4 \mathrm{hr}$ later. These manipulations were chosen because noxious stimuli (e.g., Anton et al., 1991; Gieroba et al., 1994; Carstens et al., 1995; Hathaway et al., 1995; Bellavance and Beitz, 1996) and LPS (Wan et al., 1994) elicit robust FLI within circumscribed regions of the brainstem.

For acrolein-perfused brains, the tissue was first incubated in $1 \%$ sodium borohydride for 20 min to prevent aldehyde cross-linking from causing nonspecific staining and then rinsed in PBS for $40 \mathrm{~min}$. All tissue was then incubated in $10 \%$ sheep serum in PBS for $1 \mathrm{hr}$ at room temperature. After rinsing in PBS for $30 \mathrm{~min}$, the tissue was incubated in rabbit polyclonal antibody (Santa Cruz Biotechnology, Inc., Santa Cruz, CA) diluted to a concentration of 1:6000 in PBS containing 0.4\% Triton X-100 for approximately $66 \mathrm{hr}$ at $4^{\circ} \mathrm{C}$. Several sections were separated before the primary antibody incubation and placed only into the PBS- $0.4 \%$ Triton X-100 solution to serve as a negative control. No FLI was observed in the negative control tissue. After primary antibody incubation, the sections were rinsed in PBS for $30 \mathrm{~min}$. The tissue was then incubated in biotinylated anti-rabbit IgG (Vector Laboratories, Inc., Burlingame, CA) diluted to $1: 600$ in PBS- $0.4 \%$ Triton X-100 containing $0.1 \%$ bovine serum albumin (BSA), rinsed in PBS for 30 min, and incubated in an avidin-biotin-peroxidase complex (Vector Laboratories, Elite kit; $5 \mu \mathrm{l} / \mathrm{ml}$ ) containing $0.1 \%$ BSA for $1 \mathrm{hr}$. After rinsing in $\mathrm{PB}$ for $15 \mathrm{~min}$, the tissue was incubated in a $\mathrm{PB}$ solution containing $0.05 \% \mathrm{DAB}(0.5 \mathrm{mg} / \mathrm{ml})$ and $1 \%$ nickel ammonium sulfate for $15 \mathrm{~min}$. At this time, $10 \mu \mathrm{l}$ of $30 \% \mathrm{H}_{2} \mathrm{O}_{2}$ was added to this solution for a final reaction lasting 3-5 min. To minimize the fading of FG, the tissue was monitored closely at this stage, and the reaction was stopped after the detection of the brown-black reaction product within cell nuclei denoting the presence of FLI. The tissue went through final rinses in 0.1 and $0.05 \mathrm{M} \mathrm{PB}$. After mounting onto gelatin-coated slides, sections were air dried overnight, dehydrated through ascending concentrations of alcohols, cleared with xylene, and coverslipped with cytoseal (Stephens Scientific, Riverdale, NJ).

\section{Data analysis}

FLI and retrograde label distribution. Sections were examined under a light microscope, also equipped for epifluorescence illumination, at 200-400× to identify nuclei expressing FLI, FG-labeled, and double-labeled neurons. The filter set used to visualize the FG fluorescence had a UV2A 330-380 nm emission filter, $400 \mathrm{~nm}$ Dichroic mirror, and a $420 \mathrm{~nm}$ barrier filter. FLI neurons were plotted as either "moderately" or "intensely" labeled. The criterion for an intensely labeled neuron was a brown-black to black reaction product within the cell nucleus. All other variations of brown reaction product were classified as moderately labeled. Statistical analyses were carried out for the total of both moderate and intense FLI neurons, unless otherwise noted.

This classification of FLI neurons into intense or moderate categories was verified by measuring the optical density of a random number of FLI neurons from two levels of the RF in each of two representative quinine, sucrose, and unstimulated animals using an image analysis system (Neu- 
rolucida; MicroBrightfield, Inc., Colchester, VT). Only cases with similar background optical density measurements were chosen. This was done to minimize the chance that differences in optical densities of FLI neurons were a result of differences in immunohistochemical staining, which would result in varying background levels. For this analysis, a $100 \times 100$ $\mu \mathrm{m}$ grid was overlaid on a section of the RF, and the gray scale values [ranging from 0 (black) to 255 (white)] for the nuclei lying within several randomly chosen grids were obtained and used as an index of optical density. For each of the six cases, gray scale values for FLI neurons were normalized to the most intensely stained neuron encountered from that case which was assigned a value of 100 . Collapsing across the six cases, neurons qualitatively classified as intensely labeled (mean normalized value, $119.8 \pm 19.8)$ were significantly darker $(t$ test, $p<0.001)$ than moderately labeled neurons (mean normalized value, $158.3 \pm 27.3$ ).

Under blind conditions, all FLI, FG-labeled, and double-labeled neurons were plotted on the side of the brain ipsilateral to intraoral stimulus delivery throughout seven anteroposterior levels of the RF with the aid of a video camera attached to the microscope and specialized software (Neurolucida: MicroBrightfield, Inc., Colchester, VT). The first section plotted was at the level of obex (level 0), and the remaining sections were spaced at approximate $0.5 \mathrm{~mm}$ intervals rostral to obex. At each level, the $\mathrm{RF}$ was further parcellated into anatomically defined subdivisions based on the nomenclature of Paxinos and Watson (1986). The subdivisions included the dorsal medullary reticular nucleus (MdD), ventral medullary reticular nucleus (MdV), parvocellular reticular nucleus (PCRt), intermediate reticular nucleus (IRt), gigantocellular reticular nucleus (Gi), and ventrolateral medulla (VLM). In this study, the VLM includes both the caudoventrolateral and rostroventrolateral reticular nuclei. Some subdivisions were further divided along the mediolateral (ML) and dorsoventral (DV) axes, producing quadrants so that the pattern of FLI label within a subdivision could be compared between groups.

Repeated measures ANOVAs were used to compare the mean number of FLI neurons between groups across anteroposterior levels and subdivisions of the RF, as well as within the ML and DV halves of certain subdivisions ( $n=18$ animals). When appropriate, post hoc comparisons between treatment means for all ANOVAs were made using the Tukey honest significant difference test. $t$ tests were used to compare the mean number of neurons double labeled with FLI and FG between stimulus conditions ( $n=9$ animals). For all analyses, $p<0.05$ was considered significant. $p$ values between 0.05 and 0.15 were noted as trends.

FLI and oromotor behavior correlation. The videotaped responses of seven quinine-stimulated rats were scanned at slow speed to obtain the number of gapes and passive rejections over the entire test session. This includes three animals not used for the FLI quantification. The responses of a subset of animals $(n=2)$ were counted independently by the experimenter and a person blind to the experimental procedures, and the results were averaged. Each passive rejection was counted as an all-ornone response and is defined as fluid accumulation and subsequent drip from the mouth accompanied by no oromotor or somatic responses to the infusion. The relationship between the total number of gapes or passive rejections and the total number of FLI neurons within the RF for each rat was then determined using correlational analysis ( $n=7$ animals). For this analysis, FLI was counted over three anteroposterior levels of the RF to obtain a sufficient representation of label.

\section{C-fos/anterograde tracing study}

\section{Surgical and testing procedures}

Similar surgical procedures as described above were carried out in this experiment, except that after intraoral cannulae insertion, rats were prepared for an injection of an anterograde tracer into the NST $(n=7)$. In brief, under Nembutal anesthesia $(50 \mathrm{mg} / \mathrm{kg}$, i.p.), rats were first fitted with two intraoral cannulae and then placed in a stereotaxic instrument. A hole was drilled into the skull at coordinates $(4.8 \mathrm{~mm}$ posterior and 1.8 $\mathrm{mm}$ lateral to lambda) to access NST for injections of the anterograde tracer biotinylated dextran (BD; Molecular Probes, Eugene, OR). A tungsten electrode was used to localize the NST by monitoring increases in neural activity after gustatory and/or tactile stimulation of the anterior or posterior tongue (Halsell et al., 1993; Travers and Norgren, 1995). The coordinates of the location of the NST then served as a guide for BD injection. A glass micropipette with a tip diameter of $25 \mu \mathrm{m}$ was filled with a $10 \%$ solution of BD dissolved in physiological saline. Evoked activity in NST, recorded through the pipette, determined the final site of injection. Once the pipette was situated in NST, approximately 50-100 nl of $\mathrm{BD}$ was pressure injected ( $20 \mathrm{psi}, 20 \mathrm{msec}$ pulses) into the area. The pipette remained in place for $5 \mathrm{~min}$ after the injection. The cannula assembly was then secured with dental acrylic, and the incision was closed with surgical staples. Recovery time, pretest adaptation procedures, and behavioral testing procedures were the same as described above.

\section{Immunohistochemistry}

Tissue was simultaneously processed for BD and FLI using the procedures described above.

\section{Data analysis}

Anterograde fibers showing axonal varicosities and FLI neurons were plotted at four of the seven anteroposterior levels of the RF at $400 \times$ using the Neurolucida software program. These data were plotted to determine the degree of overlap between FLI neurons and anterograde projections from the NST within the RF.

\section{RESULTS}

\section{Distribution of FLI neurons within the brainstem}

FLI was observed in the brainstem in all three stimulus conditions. Because the number of FLI neurons did not differ between acrolein-perfused and nonacrolein-perfused tissue in each group (quinine, $t(4)=1.31 ; p=0.26$; sucrose, $t(4)=0.86 ; p=0.44$; unstimulated, $t(4)=1.48, p=0.21$ ), the data were collapsed for analysis. In addition, although bilateral FLI label was observed, counts were made on the side of the brain ipsilateral to stimulus delivery.

Figure 1 shows the distribution of FLI at two levels of the medulla for a representative quinine, sucrose, and unstimulated case: one at the level of the area postrema (level 1, $0.48 \mathrm{~mm}$ anterior to obex) and the other through the rostral medulla (level 4, $1.92 \mathrm{~mm}$ anterior to obex). Although the present study focused on quantifying the distribution of FLI neurons between groups in the RF, differences were apparent in other structures, notably the NST. Sucrose and quinine stimulation resulted in a differential distribution of label within the rostral NST, in addition to eliciting more FLI compared with the unstimulated group (Fig. 1A). The FLI in the quinine group was clustered more medially, whereas the FLI within the sucrose group was more evenly distributed mediolaterally (Fig. 1 $A$ ). In the caudal NST (Fig. 1B), sucrose elicited more FLI compared with both the unstimulated and quinine groups. These findings corroborate those in a previous study from this laboratory (Harrer and Travers, 1996). Other regions in which differences in the degree of FLI between the stimulated versus unstimulated conditions were observed but not quantified included the spinal trigeminal nucleus and area postrema (see Fig. $1 B$ ).

In other areas of the medulla, FLI neurons were consistently observed regardless of stimulus condition. These areas included vestibular nuclei (medial and spinal vestibular nuclei; see Fig. 1A) and auditory-related nuclei (e.g., dorsal and ventral cochlear nuclei; not shown). In all conditions, label was also observed in the raphe nuclei (raphe obscurus and pallidus) and in and around the lateral reticular nucleus (Fig. 1). Although there seemed to be more FLI neurons in the lateral reticular nucleus of the sucrose case shown in Figure 1, this apparent difference was inconsistent across animals and conditions.

In the pons (not shown), equivalent numbers of FLI neurons were again observed in auditory-related nuclei (superior olivary complex, trapezoid nucleus, and inferior colliculus) and the pontine nuclei. Scattered label was also observed in the locus coeruleus in all conditions. In the PBN, FLI neurons were observed in the "waist" area after stimulation with either quinine or sucrose, whereas the external medial subnucleus was densely labeled only after stimulation with quinine. In contrast, FLI within the PBN of the unstimulated group was sparse and scattered. No FLI was 


\section{QUININE}

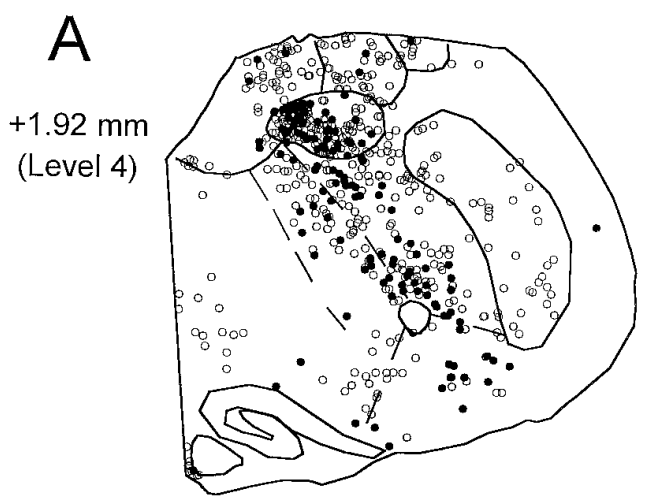

\section{SUCROSE}

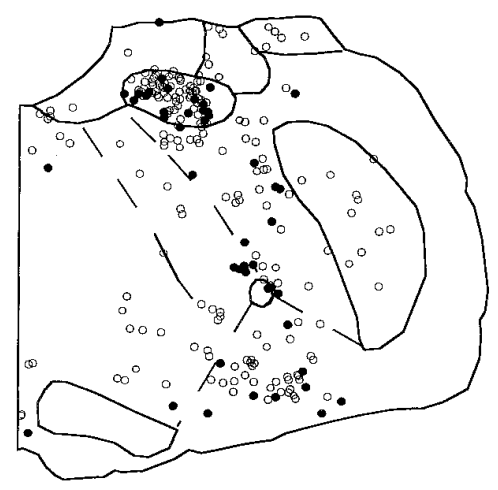

moderately-stained FLI nuclei

B

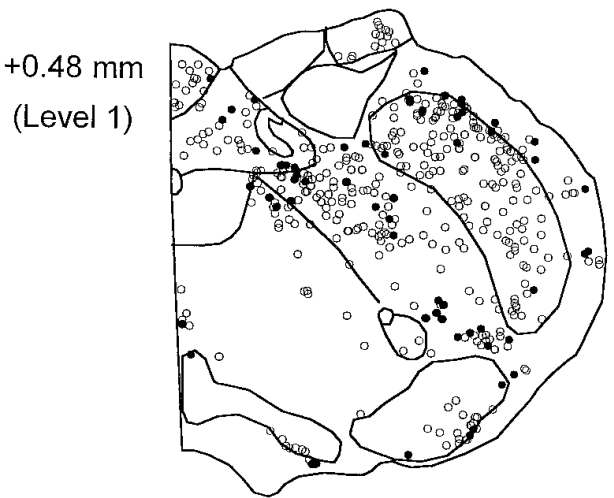

intensely-stained FLI nuclei

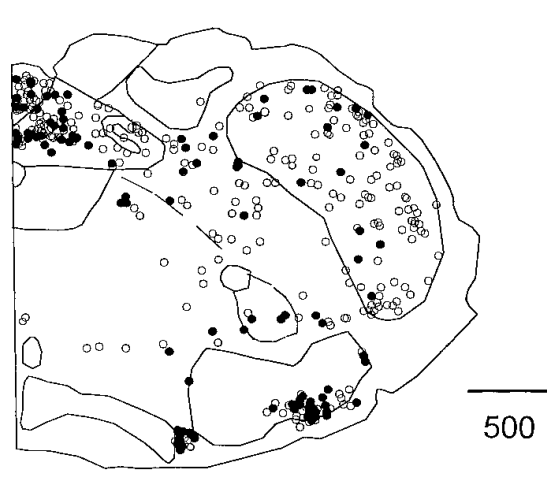

\section{UNSTIMULATED}

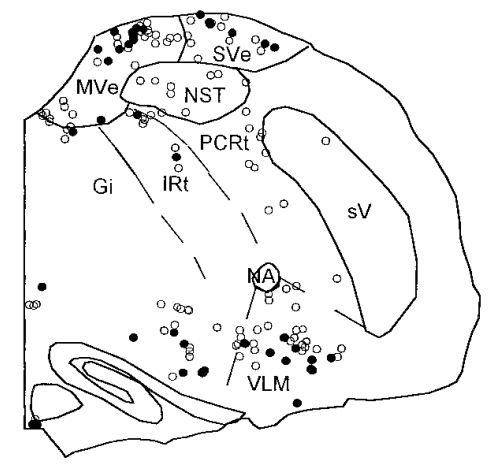

Figure 1. Distribution of FLI neurons at two anteroposterior levels of the medulla depicted in the transverse plane for a representative animal from each stimulus condition. Sections are ordered from rostral $(A$, level 4$)$ to caudal $(B$, level 1$)$. Numbers on the left indicate distance from the obex. Open circles denote moderately stained nuclei that appeared brown in color, whereas filled circles denote intensely stained nuclei that appeared black or brown-black in color. Dashed lines depict borders between reticular formation subdivisions. Abbreviations for all figures: $A P$, area postrema; $C u$, cuneate nucleus; $D C N$, dorsal cochlear nucleus; $E c u$, external cuneate nucleus; $G i$, gigantocellular reticular nucleus; $G r$, gracilis nucleus; IO, inferior olive; IRt, intermediate reticular nucleus; $L R N$, lateral reticular nucleus; $M d D$, dorsal medullary reticular nucleus; $M d V$, ventral medullary reticular nucleus; $M V e$, medial vestibular nucleus; $m V I I$, facial nucleus; $m X I I$, hypoglossal nucleus; $N A$, ambiguus nucleus; $N S T$, nucleus of the solitary tract; $P C R t$, parvocellular reticular nucleus; $p y$, pyramidal tract; $s V$, spinal trigeminal nucleus; $S V e$, spinal vestibular nucleus; and $V L M$, ventrolateral medulla.

observed in the hypoglossal, trigeminal, ambiguus, or facial motor nuclei.

\section{FLI within the RF}

Figure 2 provides a more comprehensive illustration of FLI neurons within the medullary RF for the same representative quinine and unstimulated animals in Figure 1. Because the sucrose group did not differ from the unstimulated group in terms of the total number of FLI neurons in the RF (sucrose, $X=571.67 \pm 83.65$; unstimulated, $X=491 \pm 81.15$ ), only one group (the unstimulated group) is shown. Four of the seven anteroposterior levels quantified are depicted. At the level of the obex (level 0), FLI was observed in MdV just lateral to $\mathrm{mXII}$ and extended into MdD laterally to the spinal trigeminal nucleus and ventrally toward the lateral reticular nucleus (Fig. $2 D$ ). At levels rostral to the fourth ventricle (Fig. $2 A-C$; see also Fig. $1 A$ ), FLI in the quinine group extended in a continuous band throughout the PCRt and IRt from the ventral surface of the NST to regions lateral and ventral to nucleus ambiguus, i.e., the VLM subdivision. This "band" of FLI label can also be seen in the photomicrograph in Figure 3. Compared with the quinine group, this general pattern of FLI within the PCRt and IRt was observed to a lesser degree in the sucrose group (see Fig. $1 A$ ) but was far less apparent in the unstimulated group. In all groups, scattered label was also observed in nucleus gigantocellularis (Gi) (Fig. $2 A-C$ ). Although FLI neurons were observed throughout the medullary RF in all three stimulus conditions, the quinine group had an overall larger number of both moderate and intense FLI neurons (see below).

\section{Effects of stimulus condition on FLI}

As evident in Figure 2, FLI was not distributed evenly throughout the RF. Thus, comparisons of the mean number of FLI neurons between stimulus conditions were made across the anteroposterior axis of the medullary RF, as well as across specific RF subdivisions. When the mean number of FLI neurons across seven anteroposterior levels of the RF was counted, the quinine group 

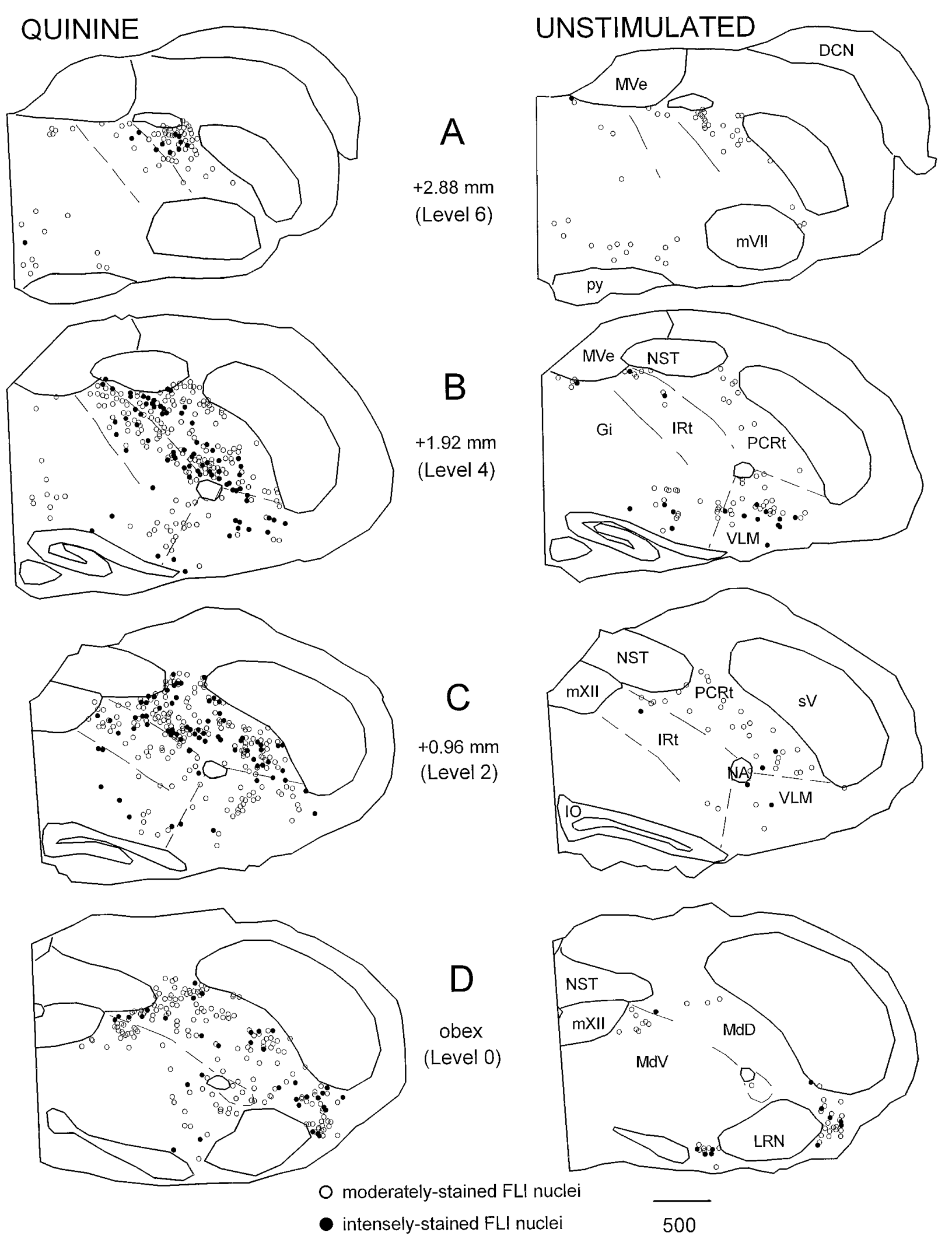

Figure 2. Distribution of FLI neurons throughout the medullary reticular formation for the same representative quinine and unstimulated animals in Figure 1. Only FLI neurons within the RF are plotted. Sections are ordered from rostral $(A$, level 6$)$ to caudal $(D$, level 0$)$. Numbers indicate distance from obex. Open circles denote moderately stained nuclei that appeared brown in color, whereas filled circles denote intensely stained nuclei that appeared black or brown-black in color. Dashed lines depict borders between reticular formation subdivisions. 


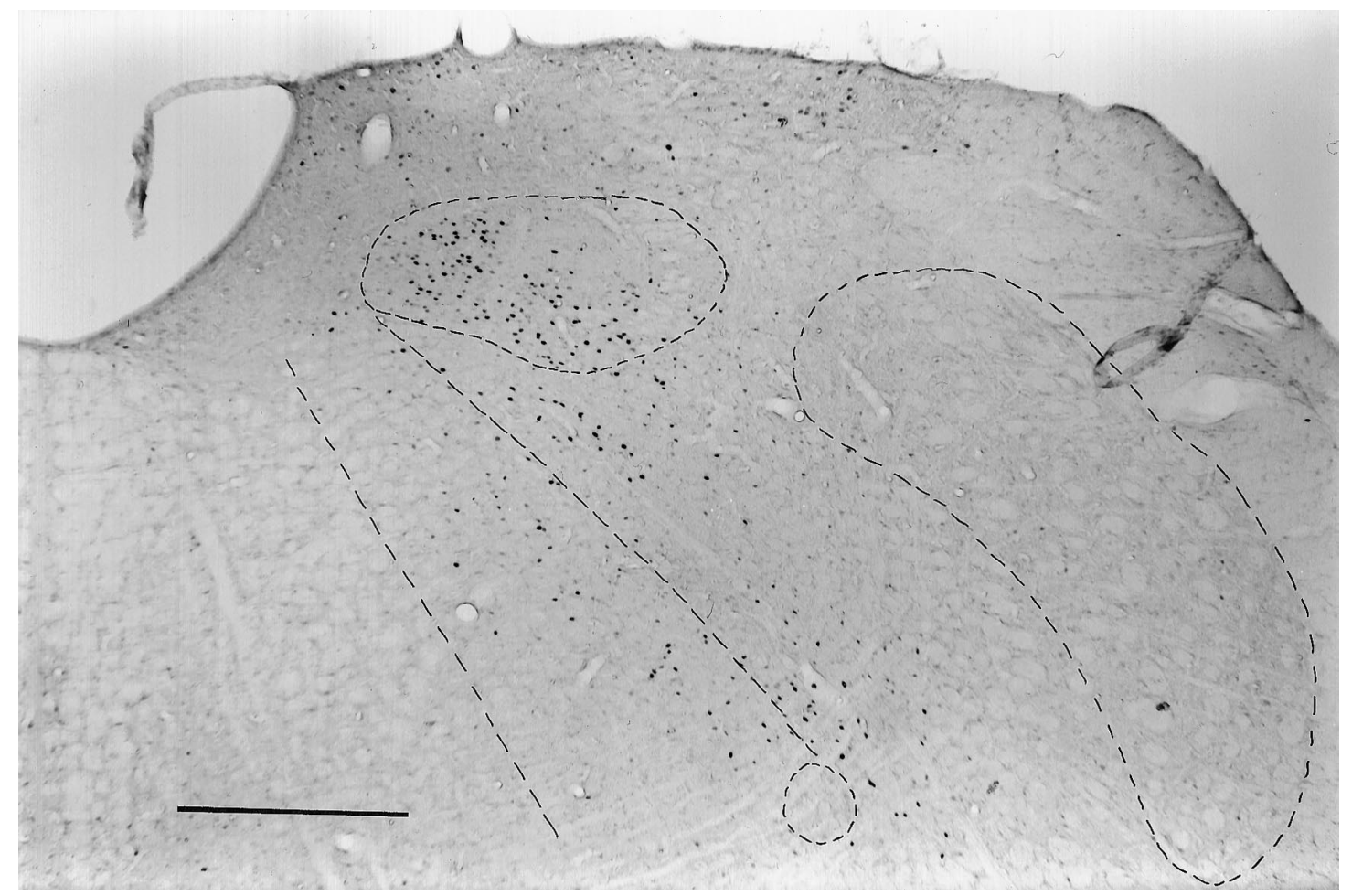

Figure 3. Low-power photomicrograph of the distinct pattern of FLI in the medulla at level 4 for the same quinine animal in Figures 1 and 2 . The nucleus of the solitary tract, spinal trigeminal nucleus, and nucleus ambiguus are outlined as well as the borders between PCRt, IRt, and Gi. Scale bar, $500 \mu \mathrm{m}$.

had approximately twice as many FLI neurons compared with the sucrose and unstimulated groups at levels $0-5$ (Fig. 4A). At level 6 , FLI decreases for all groups. A repeated measures ANOVA comparing the mean number of FLI neurons across these levels of the RF revealed a significant main effect for stimulus condition $\left[F_{(2,15)}=11.81 ; p<0.01\right]$ and level $\left[F_{(6,90)}=16.53 ; p<0.001\right]$ and a significant stimulus condition $\times$ level interaction $\left[F_{(12,90)}=\right.$ 2.17; $p<0.05]$. Post hoc analysis indicated that the mean number of FLI neurons in the quinine group was significantly greater than the mean number of FLI neurons in both the sucrose and unstimulated groups at levels $0-5$ (Fig. $4 A$ ). Although the quinine group had more FLI neurons at level 6 compared with the other two groups, this difference was not significant. In addition, although sucrose consistently labeled more neurons than the unstimulated group by an average of $14 \%$, no statistical differences between these conditions were evident.

A similar analysis was performed to compare the number of FLI neurons in each of six RF subdivisions (Fig. 4B). Quinine elicited significantly more FLI in three subdivisions compared with the sucrose and unstimulated conditions. The greatest difference was observed in PCRt in which the quinine group had almost three times more FLI neurons compared with the other groups. A repeated measures ANOVA indicated a significant main effect for subdivision $\left[F_{(5,75)}=50.60 ; p<0.001\right]$ and a significant stimulus condition $\times$ subdivision interaction $\left[F_{(10,75)}=\right.$ 13.45; $p<0.001]$. Post hoc analysis revealed that the quinine group exhibited a significantly greater number of FLI neurons than both the sucrose and unstimulated groups in MdD, PCRt, and IRt (Fig. 4B). In VLM, there was only a trend toward a greater number of FLI neurons in the quinine group compared with the unstimulated group $(p=0.10)$. When only intense FLI neurons were counted in VLM, however, there was a statistically significant difference between the quinine and unstimulated groups $(p=0.02)$. Again, no significant differences between the sucrose and the unstimulated groups were found, although sucrose elicited more FLI in VLM compared with the unstimulated condition (Fig. 4B).

\section{Pattern of label within RF subdivisions}

Visual inspection of the tissue suggested that FLI neurons were differentially distributed within certain subdivisions of the RF depending on stimulus condition. For example, FLI seemed to be clustered more laterally in PCRt in the unstimulated and sucrose groups compared with the quinine group (see Figs. 1 and 2). Thus, the pattern of label within RF subdivisions was further analyzed to determine whether the anatomical location of FLI within a subdivision differed significantly between groups. The analysis was restricted to those regions in which statistically significant differences between the quinine group and the other groups were observed (MdD, PCRt, and IRt). For this analysis, the three subdivisions were equally divided along the mediolateral (ML) and dorsoventral (DV) axes producing quadrants. Within each subdivision, a repeated measures ANOVA compared the mean number of FLI neurons across the ML and DV halves, as well as across each of the four quadrants.

In $\mathrm{MdD}$, a significant stimulus condition $\times \mathrm{DV}$ axis interaction $\left[F_{(2,15)}=10.42 ; p=0.001\right]$ indicated that the quinine group exhibited a greater number of FLI neurons in the dorsal half compared with the ventral half, whereas the sucrose and unstimulated groups exhibited an equal distribution of FLI neurons along the dorsal and ventral halves. Furthermore, comparisons of the distribution of FLI neurons across each of the four quadrants of MdD revealed that the FLI in the quinine group was specifically clustered in the dorsomedial quadrant $\left[F_{(6,45)}=3.07 ; p<0.05\right]$. 
A

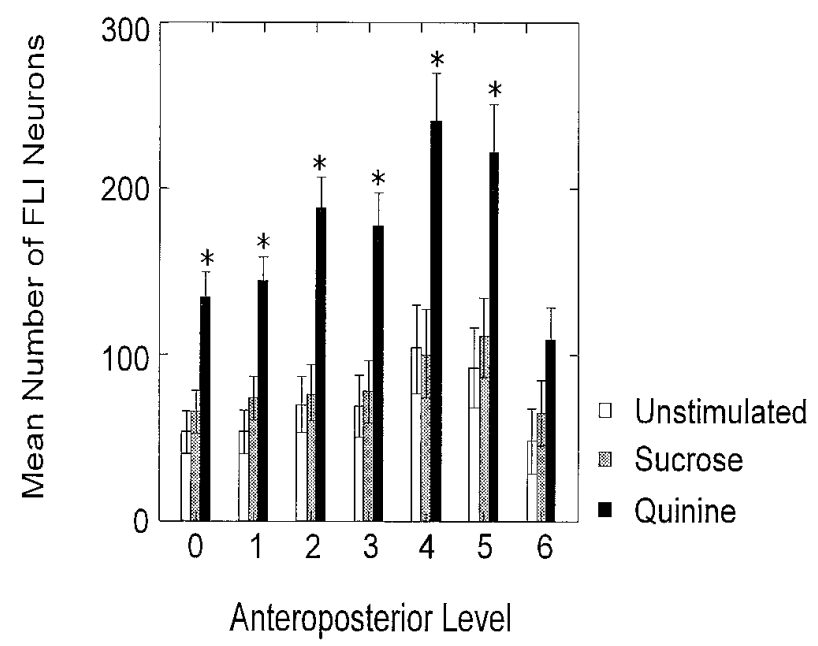

B

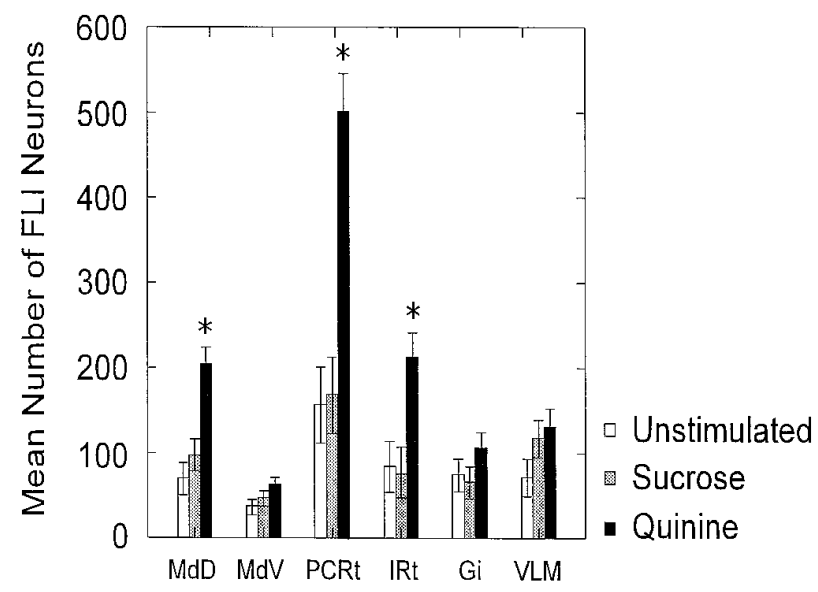

Subdivision

Figure 4. Mean ( \pm SEM) number of FLI neurons $(A)$ across seven anteroposterior levels of the reticular formation from caudal (level 0 , obex) to rostral (level 6) and $(B)$ across six reticular formation subdivisions. Asterisks indicate significant differences between the quinine group and the other two groups.

In PCRt, a stimulus condition $\times$ ML axis interaction was significant at two rostral levels of the RF: level $4\left[F_{(2,15)}=8.73 ; p<\right.$ $0.01]$ and level $5\left[F_{(2,15)}=4.10 ; p<0.05\right]$. Post hoc analysis revealed that the quinine group exhibited a greater number of FLI neurons in the medial half of PCRt compared with the lateral half at these levels, whereas the sucrose and unstimulated groups exhibited an equal distribution of FLI neurons along the medial and lateral halves. Furthermore, comparisons of the distribution of FLI neurons across each quadrant of PCRt revealed a specific dorsomedial cluster at levels $4\left[F_{(6,45)}=4.14 ; p<0.01\right]$ and 5 $\left[F_{(6,45)}=3.35 ; p<0.01\right]$ in the quinine group. In IRt, a significant stimulus condition $\times$ ML axis interaction $\left[F_{(2,15)}=12.85 ; p<\right.$ 0.01] indicated that FLI neurons in the quinine group were distributed more in the lateral half compared with the medial half. Furthermore, comparisons of the distribution of FLI neurons across each quadrant of IRt revealed specific dorsolateral (level 5) and ventrolateral (levels 4 and 5) clusters $\left[F_{(6,45)}=7.47 ; p<\right.$ $0.001]$ in the quinine group.

\section{Correlation between FLI in the RF and oromotor behavior after quinine stimulation}

As expected from the numerous studies using intraoral taste stimulation, rats in each group displayed distinct behaviors depending on the stimulus condition. Rats that received sucrose or quinine displayed stereotypical oromotor ingestive and rejection behaviors, respectively (Grill and Norgren, 1978a; Travers and Norgren, 1986). Ingestive behaviors included licks and lateral tongue protrusions; rejection behaviors consisted of gapes, chin rubs, passive rejections, and various somatic responses including head shakes, forelimb flails, and grooming. It was of interest that rats demonstrated these rejection responses to varying degrees. Some rats reacted more "actively" to quinine and thus showed many gapes (range, 212-633) and chin rubs (range, 75-188) and fewer passive rejections (range, 14-82 of 144 possible). Other rats were less active and showed more passive rejections (range, 101108 of 144 possible) than gapes (range, 44-83) or chin rubs (range, 4-46). These interanimal differences in the amount of oromotor responses to quinine have been reported previously (Parker, 1994). In addition to the oromotor and somatic rejection responses, rats also reared and moved around the testing chamber extensively, indicative of an agitated state. Rats that demonstrated more passive rejections than gapes also tended to show less rearing and locomotion, although this was not the case for one rat that moved around the testing chamber (almost) continuously. Although not directly measured, all rats that received quinine also appeared to drool throughout the test session, a condition not observed with the sucrose or unstimulated groups. The unstimulated controls did not show any distinct oromotor behaviors. Instead, the behaviors commonly observed were tooth grinding and locomotion, events that occurred briefly and sporadically throughout the test session. Overall, all rats in this group seemed to be asleep or at rest for most of the test session.

Because quinine-stimulated rats varied in terms of the number of active (gapes) and passive rejection responses, correlations between total FLI and these behaviors were made. These correlations could provide some insight as to whether the number of FLI neurons in the RF was related to the degree of oromotor activity. Four of six of the animals used for the anatomical analyses were used in this analysis, as well as three additional animals for which behavioral observations were available. Only five of seven animals had sufficient information for performing the correlations between FLI and passive rejections. Scatter plots of the number of FLI neurons and the number of gapes $(n=7)$ and passive rejections $(n=5)$ indicate a strong correlation (Fig. 5). Total FLI was positively correlated with gapes $(r=0.90 ; p<0.01)$ and negatively correlated with passive rejections $(r=-0.91 ; p<$ 0.05), suggesting that more FLI is elicited in the RF of rats that could be categorized as "active" rejectors (i.e., more gapes relative to passive rejections). When the relationship between rejection behavior and FLI was analyzed within each RF subdivision, there were significant positive correlations between gapes and FLI within $\mathrm{MdV}(r=0.82 ; p<0.05)$, IRt $(r=0.94 ; p<0.01), \mathrm{Gi}(r=$ 0.97; $p<0.001)$, and VLM $(r=0.84 ; p<0.05)$. Significant negative correlations were found between passive rejections and FLI within IRt $(r=-0.91 ; p<0.05)$, Gi $(r=-0.95 ; p<0.05)$, and $\operatorname{VLM}(r=-0.96 ; p<0.01)$. 

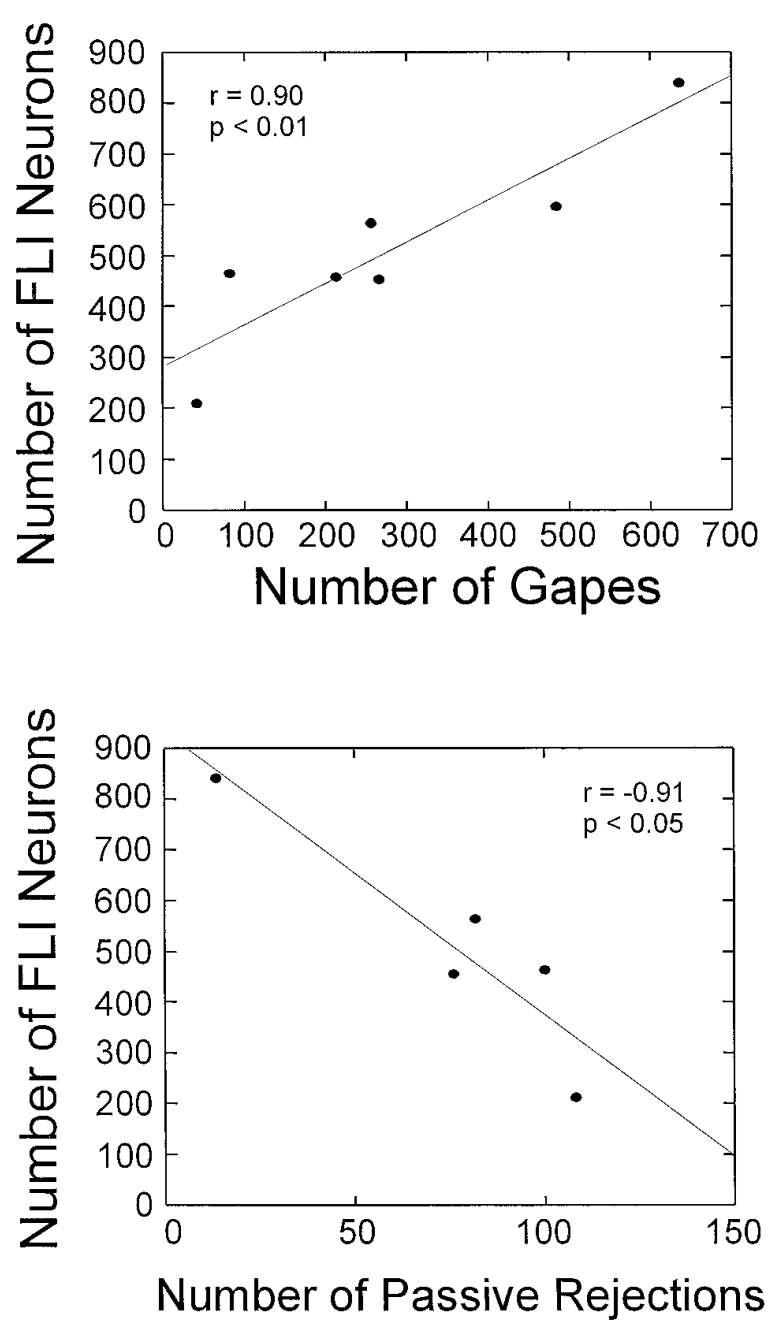

Figure 5. Scatter plots and linear regression comparing the number of FLI neurons in the reticular formation and the number of gapes (top) or number of passive rejections (bottom). Each symbol represents one animal.

\section{Colocalization of FLI and RF projection neurons}

To gain further insight into the possible functional significance of the FLI, the connectivity between RF neurons exhibiting FLI and mXII was examined. A total of 18 animals were injected with FG into $\mathrm{mXII}$, of which 9 provided useful data. The distribution of retrogradely labeled neurons in the RF after the two FG injections into the trigeminal motor nucleus was qualitatively similar to the distribution after $\mathrm{mXII}$ injections. Thus, only the results of the $\mathrm{mXII}$ injections are discussed. A distinct pattern emerged when the distributions of FLI, FG-labeled, and double-labeled neurons were compared. These distributions are plotted in Figure 6 for a representative quinine animal. In rostral levels of the medulla (Fig. 6A,B), the FG distribution (filled triangles) was primarily located in IRt. This distribution was somewhat segregated from the FLI (open circles), which was more densely concentrated in PCRt (as previously described), although there was certainly overlap between FLI and FG neurons within IRt. In caudal levels of the medulla (Fig. 6C), FG neurons were distributed mainly within the dorsal half of $\mathrm{MdV}$ and $\mathrm{MdD}$, spanning an area from just lateral to $\mathrm{mXII}$ to the spinal trigeminal nucleus. Although there was overlap between FG and FLI within these areas, FLI neurons extended more ventrally than FG neurons. Similar pat- terns were also observed in the sucrose and unstimulated groups. In all groups, most of the double-labeled neurons (shaded squares) were located in IRt, with fewer in PCRt, MdD, MdV, and along the lateral edge of mXII (see Fig. 6). Figure 7 depicts some examples of FG-labeled and double-labeled neurons within the IRt of the same case shown in Figure 6.

Double-labeled neurons were not evenly distributed along the rostrocaudal axis of the RF. Figure 8 is a graph of the mean number of double-labeled neurons distributed across seven anteroposterior levels of the RF. All groups showed a similar pattern in the distribution of double-labeled neurons with a peak at level 3. This pattern was similar to the distribution of FG neurons that also peaked at level 3 for all groups (not shown). This pattern did not, however, parallel the distribution of FLI, which instead showed a peak further rostrally at levels 4 and 5 (see Fig. $4 A$ ).

Although the quinine group had approximately $2.5 \mathrm{x}$ more FLI neurons in the RF than the sucrose or unstimulated groups, all groups had an equivalent number of $\mathrm{FG}$ neurons $\left[F_{(2,6)}=0.71\right.$; $p=0.53]$. Thus, if the number of double-labeled neurons varies simply as a function of differences in the number of FLI neurons, then the percentage of total FLI neurons double labeled would be equivalent for all groups. This was not the case. Rather, quininestimulated animals exhibited disproportionate numbers of double-labeled neurons. Instead of equal proportions of FLI neurons double-labeled across groups, an average of $3.73 \%$ of total FLI neurons were double labeled after quinine stimulation compared with the sucrose $(X=2.44 \%)$ and unstimulated $(X=$ $1.40 \%$ ) groups (Fig. 9, shaded bars). $t$ tests comparing these means indicated that the quinine group had a significantly higher percentage of double-labeled neurons compared with the unstimulated group $(p<0.05)$ but not the sucrose group. The sucrose group was not significantly different from the unstimulated group. Moreover, the percentage of FG neurons double labeled for the quinine group should be 2.5 times higher than the other groups, reflecting the increase in the number of quinine-induced FLI neurons. Instead, there were approximately seven times as many FG neurons double labeled in the quinine group compared with the sucrose group (quinine, $X=17.90 \%$, vs sucrose, $X=2.50 \%$ ) and 12 times as many compared with the unstimulated group (quinine, $X=17.90 \%$, vs unstimulated, $X=1.52 \%$; see Fig. 9, filled bars).

\section{Colocalization of FLI and NST anterograde label}

The distribution of FLI neurons within the RF was compared with the distribution of anterograde projections from NST to the RF to determine whether these FLI neurons in the RF may receive gustatory or oral somatosensory afferent input. A total of seven animals were injected with BD into the rostral NST, of which four provided useful data. Two of the four injections were made into NST at sites in which responses to stimulation of the anterior tongue with a taste mixture were recorded. The other two injections were made into NST at sites in which responses to tactile stimulation of the posterior tongue (foliate papillae) were recorded. Figure 10 shows a plot of a representative BD injection into a foliate tactile-responsive site of the NST and FLI after stimulation with quinine. The injection site (Fig. 10B, black region) was centered within the ventral subdivision of the NST approximately $1.92 \mathrm{~mm}$ anterior to the obex (level 4). The injection filled the NST at this level and spread slightly ventrally around a blood vessel (Fig. 10B, light gray-shaded region). Labeled fibers were seen in several areas of the medulla both rostral and 


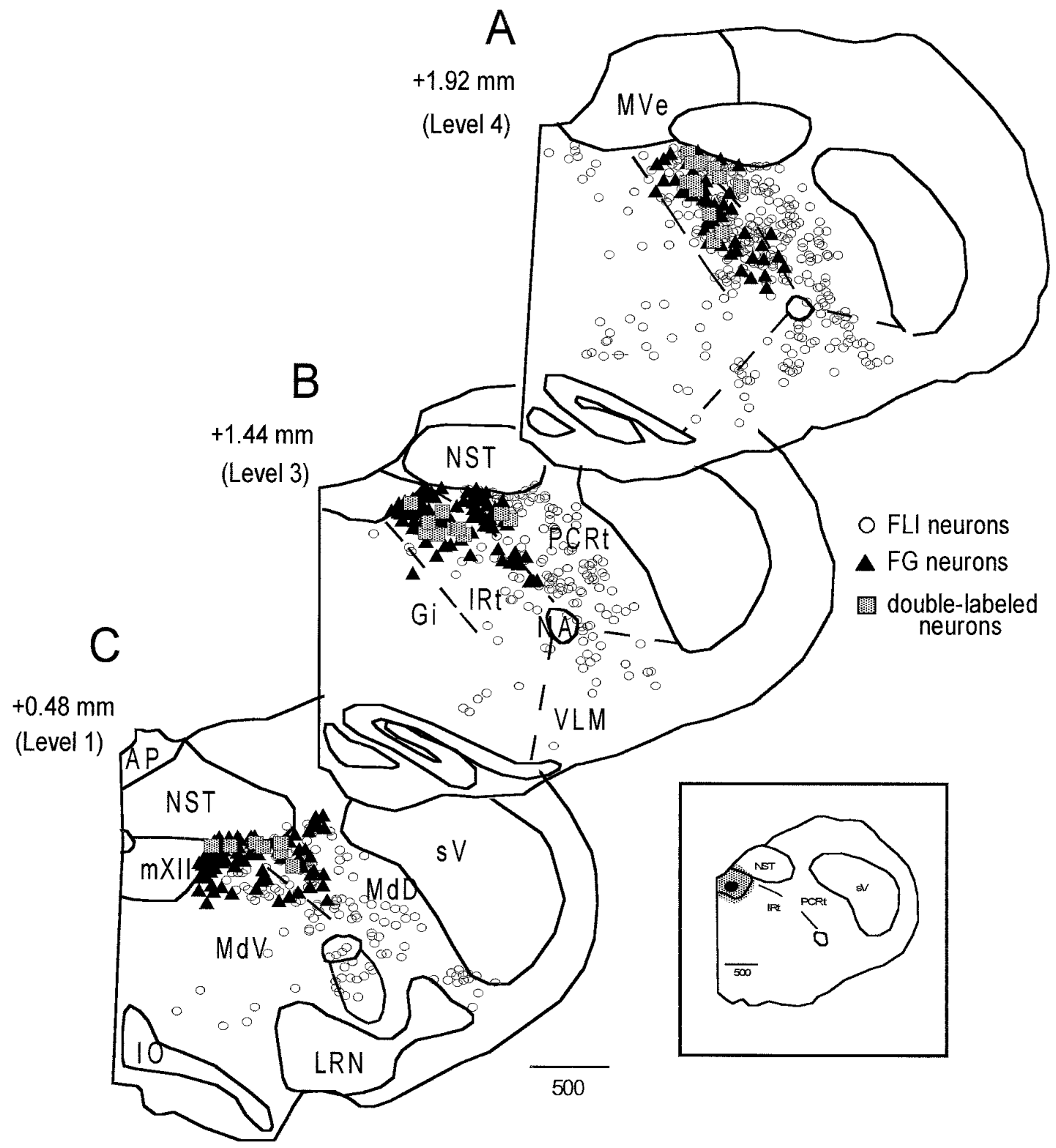

Figure 6. Distribution of FLI (open circles), FG-labeled ( filled triangles), and double-labeled (shaded squares) neurons throughout the medullary reticular formation for a representative quinine animal. Sections are ordered from rostral $(A)$ to caudal $(C)$. Numbers on the left indicate distance from obex. The inset depicts the location of the FG injection site within the hypoglossal nucleus.

caudal to the injection site. At levels rostral to the injection site (level 6, Fig. 10A), label was observed within NST and throughout IRt and PCRt extending ventrally to the facial nucleus. At the level of the injection site (Fig. 10B) and caudal to it (level 2, Fig. $10 C$ ), labeled fibers extended throughout PCRt and IRt ventrally toward nucleus ambiguus. Some label also extended into VLM. In the caudal medulla, at the level of the obex (Fig. 10D), label was observed mainly in the dorsal half of $\mathrm{MdD}$ and $\mathrm{MdV}$ with less in the ventral regions of MdD and MdV. Label was also observed directly within mXII (see Fig. 10C,D). Intranuclear label within caudal areas of the NST tended to be clustered in medial and ventrolateral subdivisions (Fig. 10C,D).

At all levels, the distribution of anterograde label was intermingled with the distribution of FLI neurons, both within the NST and RF. At high magnification, axonal varicosities were observed in close apposition to many FLI nuclei, suggesting the possibility of direct connections with these cells (Fig. 11). However, there were regions containing anterograde label with a lack of FLI neurons and vice versa. The areas in which FLI was not colocalized with anterograde label tended to be in more medial and ventral regions of the RF.

One small BD injection site was centered in the rostral central subnucleus of the NST, at a foliate tactile-responsive site, with little to no spread into the ventral subdivision (not shown). In this case, anterograde label was observed in NST rostral and caudal to the injection but was not as dense in NST caudal to the fourth ventricle. Also, little to no label was observed in mXII or the medullary RF. Anterograde fibers were observed, however, in gustatory regions of the PBN. These results confirm the findings of Halsell et al. (1996), in which the majority of descending projections from the NST to the medullary RF arise from neurons located mainly in the ventral subnucleus of the NST, whereas the majority of ascending projec- 
Figure 7. Photomicrograph of Fluorogoldlabeled and double-labeled neurons in IRt at level 5 from the same quinine animal in Figure 6. Arrows highlight just a few of the double-labeled neurons that can be seen in this photo. Top, Dorsal; left, medial. Scale bar, $50 \mu \mathrm{m}$.

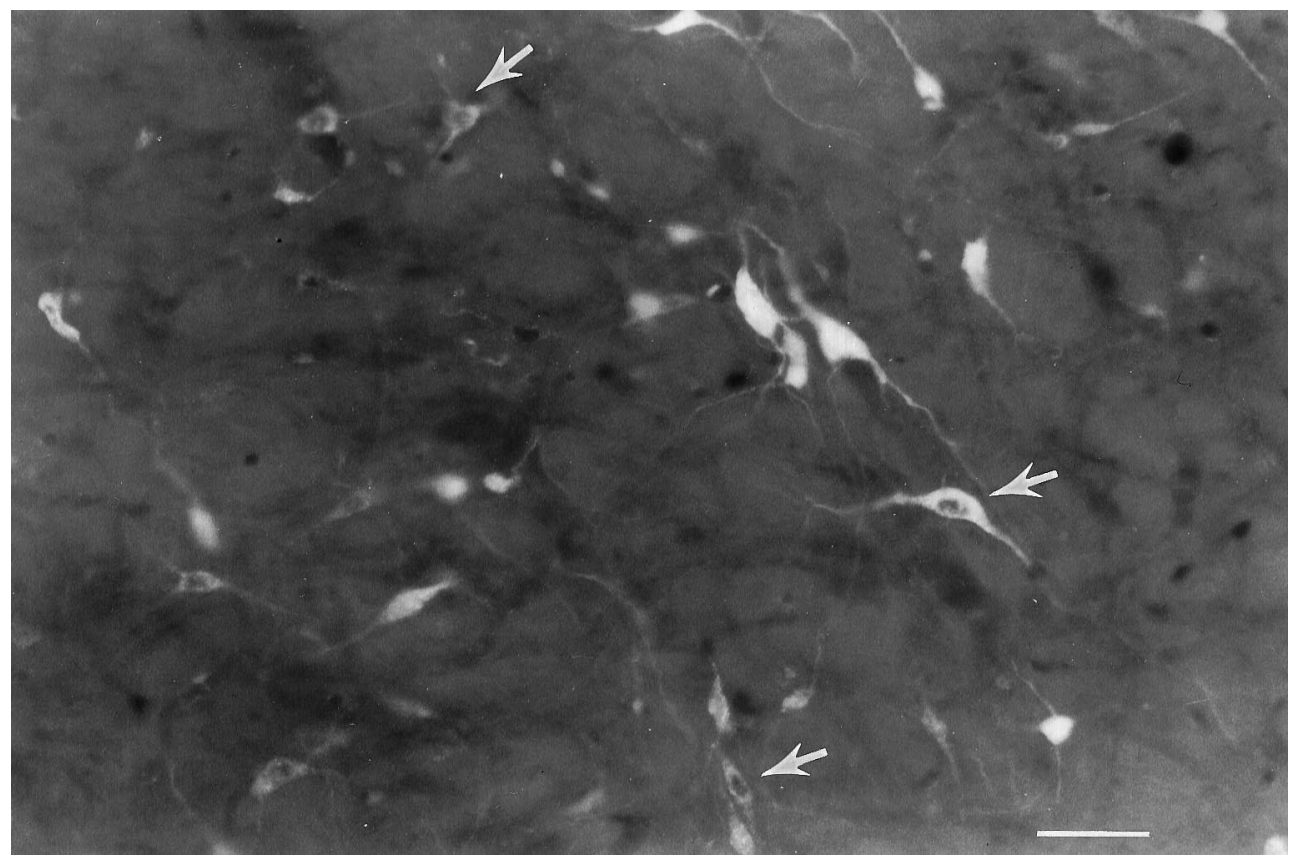

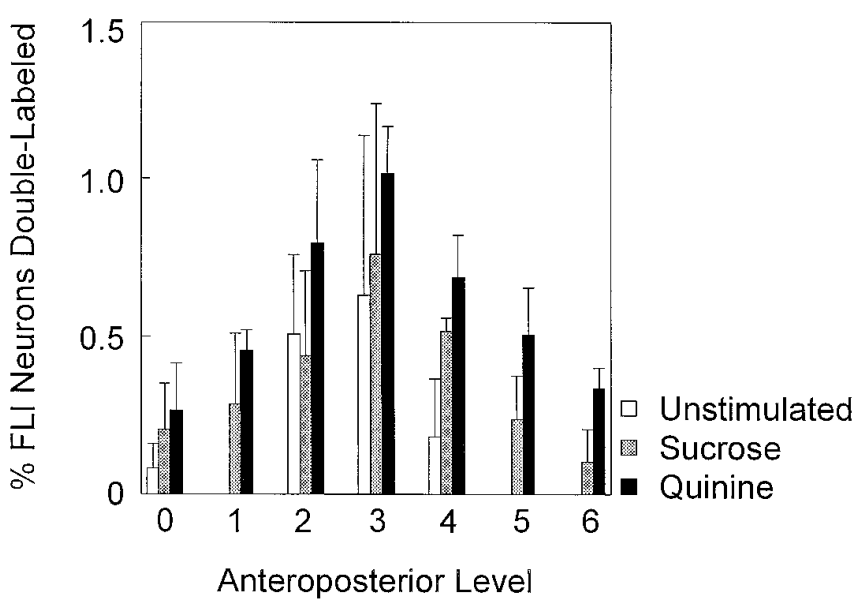

Figure 8. Mean ( \pm SEM) number of double-labeled neurons across the anteroposterior axis of the medullary reticular formation for each stimulus condition. Double-labeled neurons are expressed as a percentage of total FLI neurons.

tions from the NST to the PBN originate from neurons within the rostral central subnucleus of the NST.

\section{DISCUSSION}

\section{The pattern of quinine-elicited FLI in the brainstem}

Both sucrose and quinine stimulation resulted in the expression of Fos in brainstem gustatory nuclei (NST and PBN), confirming other reports of Fos expression in these areas after gustatory stimulation in awake rats (Yamamoto et al., 1994; Harrer and Travers, 1996). Only the rejection of quinine, however, elicited significantly more FLI neurons in RF regions implicated as interneuronal substrates in the control of oromotor reflexes (Nakamura and Katakura, 1995; Travers et al., 1997). Other studies have also reported Fos expression in interneurons associated with motor circuits controlling locomotion (Jasmin et al., 1994a; Carr et al., 1995), scratching (Barajon et al., 1992), sneezing (Wallois et al., 1995), and emesis (Miller and Ruggiero, 1994). In the present

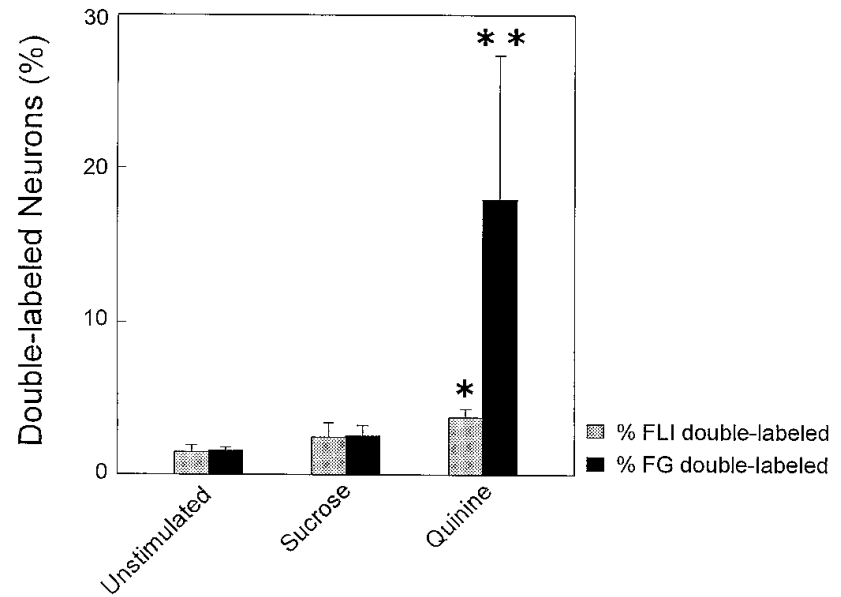

Stimulus

Figure 9. Bar graph depicting the mean ( \pm SEM) percent of FLI neurons that were double-labeled (shaded bars) and Fluorogold $(F G)$ neurons that were double-labeled ( filled bars) for each stimulus condition. *, Significant difference between the quinine group and the unstimulated group. **, Significant differences between the quinine group and the other two groups.

study, no FLI was observed in the motor limb of the circuit, i.e., the oromotor nuclei. Although most studies examining FLI in either acute anesthetized or unanesthetized preparations have also typically reported the lack of FLI within brainstem motor nuclei (Rinaman et al., 1993; Gieroba and Blessing, 1994; Li and Dampney, 1994; Yousfi-Malki and Puizillout, 1994; Hathaway et al., 1995; Wallois et al., 1995; but see Emond and Weingarten, 1995), there have been several reports of Fos expression in spinal motoneurons (Jasmin et al., 1994a; Carr et al., 1995).

In the RF, more FLI neurons after rejection of quinine were found in PCRt, IRt, and MdD compared with the sucrose and unstimulated groups. These regions are likely involved in oromotor reflex control, because they receive input from the gustatory NST and PBN (Norgren, 1978; Travers, 1988; Beckman and 

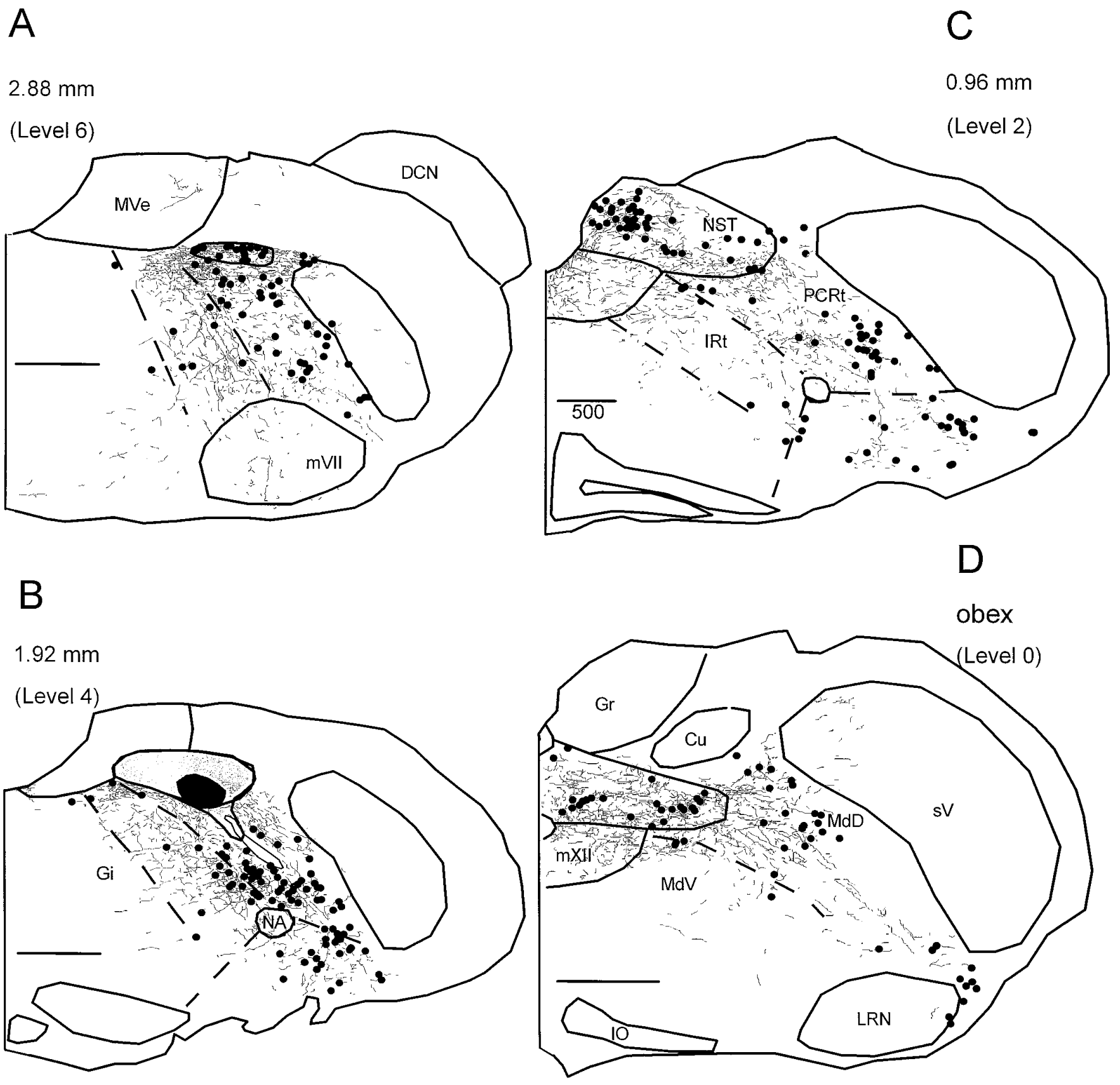

Figure 10. Distribution of FLI neurons ( filled circles) and labeled fibers throughout the medullary reticular formation of a quinine-stimulated animal. The injection of the anterograde tracer biotinylated dextran was made at a foliate tactile-responsive site in NST. The center of the injection site and spread within NST are illustrated in $B$ as a dark region surrounded by a lightly shaded region. Sections are ordered from rostral $(A$, level 6$)$ to caudal $(D$, level $0)$. Numbers indicate distance from obex.

Whitehead, 1991; Becker, 1992; Halsell et al., 1996; Karimnamazi et al., 1996), project to the oromotor nuclei (Holstege et al., 1977; Travers and Norgren, 1983; Ter Horst et al., 1991), and contain neurons that have activity specifically correlated with licks, swallows, and gapes (Siegel and Tomaszewski, 1983; Car and Amri, 1987; Jean, 1990; Amri et al., 1991; Travers and DiNardo, 1992; Karimnamazi et al., 1994). Because gapes in rats seem to be an abbreviated version of the emetic response in other species, the distinct pattern of FLI observed after quinine stimulation may represent the activation of a specific "oral rejection" circuit that is related to an emetic control circuit. In fact, the pattern of FLI in the RF observed in the present study resembles the pattern of FLI observed in cats after emesis (Miller and Ruggiero, 1994). In addition to oromotor reflex control, MdD is considered an important site for nociceptive transmission, and its connections to the oromotor nuclei may modulate orofacial reactions to pain (Bernard et al., 1990; Almeida et al., 1996). More generally, MdD may be involved in the control of behavioral reactions common to a variety of aversive stimuli.

There was a trend for an increased number of FLI neurons in VLM after stimulation with quinine and sucrose compared with the unstimulated controls, and a significant positive correlation 


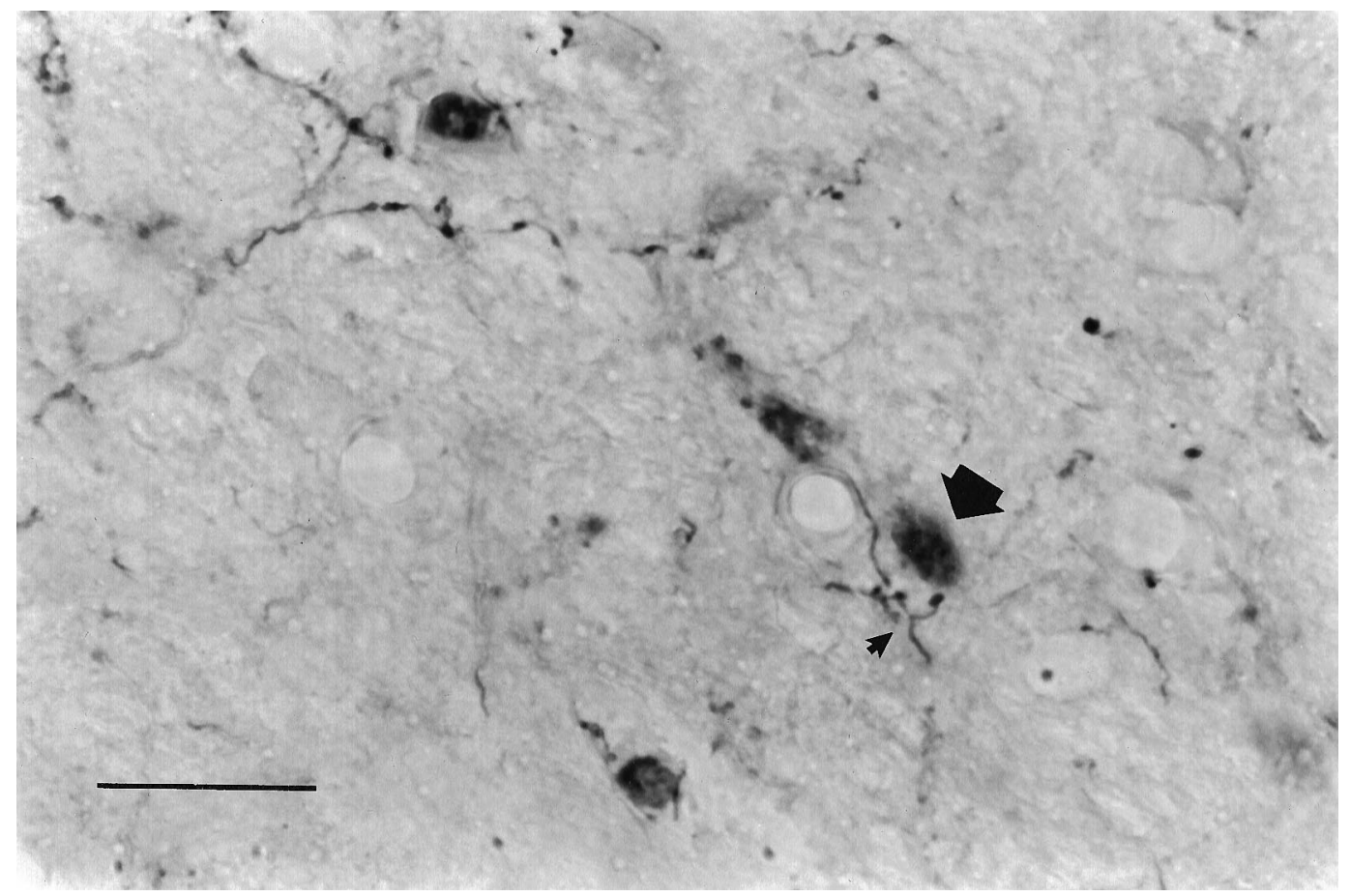

Figure 11. Photomicrograph depicting anterogradely labeled fibers in relation to FLI nuclei in PCRt at level 4 for the same animal in Figure 10. Note the labeled fiber showing axonal varicosities (small arrowhead) in close apposition to a FLI nucleus (large arrowhead). Top, Dorsal; left, medial. Scale bar, $25 \mu \mathrm{m}$.

between the number of gapes and FLI neurons in VLM. Because VLM is a region implicated in cardiovascular and respiratory control (Feldman and Ellenberger, 1988; Guyenet, 1990), the increased number of FLI neurons could reflect autonomic activation occurring during ingestion and rejection. Cardiovascular changes associated with feeding have been observed in both awake cats (Matsukawa and Ninomiya, 1987) and rats (Contreras et al., 1996).

In all groups, a distinct cluster of FLI neurons was observed just lateral to $\mathrm{mXII}$ in $\mathrm{MdV}$, an area that contains premotor neurons to mXII (Travers and Norgren, 1983; Dobbins and Feldman, 1995; Travers et al., 1995). It is unclear why the unstimulated group, in particular, showed FLI in this region, but it may reflect neural activity related to maintaining lingual tone for a patent airway (Lowe, 1981). Similar counts of FLI neurons across conditions in $\mathrm{Gi}$, a region involved in locomotor control (Perreault et al., 1994), may reflect movement in the testing chamber before and during testing that also occurred in all groups. Although portions of Gi may be crucial to the generation of ororhythmic activity in some species (Nakamura and Katakura, 1995), this role in rat is unclear (Travers et al., 1997).

\section{Quinine-elicited FLI in the RF: behavioral significance}

The observation that stimulation with quinine, but not sucrose, elicited significantly more FLI neurons in the RF may reflect the more robust motor activity associated with the rejection (gape) response. Gapes are differentiated from licks by increases in the durations and amplitudes of orolingual muscle contractions, as well as modification of the phase relationships between them (Travers and Norgren, 1986; DiNardo and Travers, 1994). In $\mathrm{mXII}$, gapes are associated with enhanced activity and shifts in the firing patterns of single neurons that are active during licking, rather than recruitment of additional motoneurons (DiNardo and
Travers, 1994). More FLI neurons within the RF after quinine stimulation, however, suggests that the "switch" in the motor pattern from ingestion to rejection is produced by the recruitment of unique RF premotor neurons. Thus, the quinine group had a disproportionately higher percentage of FLI premotor (i.e., double-labeled) neurons $(17.9 \%)$ compared with the sucrose $(2.5 \%)$ and unstimulated $(1.5 \%)$ groups, possibly reflecting an increased number of neurons needed to coordinate the sequence of complex orolingual movements coupled to somatic behaviors (e.g., chin rubs, head shakes, and forelimb flails) characteristic of rejection. In addition, although not reaching statistical significance, there was also a trend for an increase in the number of double-labeled neurons associated with an increase in the number of gapes.

Premotor recruitment as a mechanism for motor switching has been reported in other species. For example, in Xenopus embryos, two distinct rhythmic motor patterns are generated by spinal cord circuitry: swimming and struggling. Although both behaviors use the same muscles, struggling is a more robust movement than swimming. Soffe (1993) demonstrated that both behaviors are generated by a common circuit, with additional neurons recruited for struggling from within the same neuronal classes. In the rat, the recruitment of premotor neurons from the same neuronal pool is likely, because lick- and gape-responsive neurons are found within the same RF regions (Travers and DiNardo, 1992; Karimnamazi et al., 1994).

Compared with unstimulated controls, sucrose ingestion elicited a slight, but nonsignificant, increase (14\%) in the number of RF neurons exhibiting FLI. The reason for the lack of a significant difference is unclear but may reflect the absence of biochemical messengers needed for regulating Fos activation in some neurons (Dragunow and Faull, 1989). Furthermore, some brain regions do 
not express Fos under basal (Herdegen et al., 1991) or tonically active (Hoffman et al., 1994) conditions. Alternatively, if Fos expression in the RF reflects primarily motor events, licking behavior may not have reached a critical threshold necessary to express the protein. Likewise, the $14 \%$ increase in the number of FLI neurons compared with unstimulated controls may have produced a unique RF distribution that we were unable to detect. Certainly, both the pattern and number of FLI neurons in the NST were different than the unstimulated controls, indicating the overall efficacy of our stimulation protocol.

\section{Functional topography of the RF}

Although widespread increases in FLI throughout the RF were associated with quinine stimulation, the differential pattern of label within various subdivisions may reflect aspects of a functional organization of the RF. Thus, the distribution of FLI neurons in PCRt, which was also the major recipient of projections from the NST, was distinct from the distribution of mXII premotor neurons in IRt. If we postulate PCRt to IRt short axon connections, the flow of sensorimotor processing from lateral to medial is suggested (Brodal, 1957). Direct evidence for such projections, however, awaits further confirmation.

Further evidence for a mediolateral functional topography of the RF comes from the correlations between the number of FLI neurons in each RF subdivision and the degree of oromotor activity. The number of FLI neurons in IRt and Gi increased significantly as the number of gapes increased and the number of passive rejections decreased. In the more lateral RF (PCRt and $\mathrm{MdD}$ ), the correlations between oromotor behavior and FLI were nonsignificant, even though PCRt contained, overall, more FLI neurons compared with IRt and Gi. Similarly, MdD had more FLI neurons compared with Gi. Thus, the degree of FLI expressed after quinine stimulation varied consistently as a function of oromotor activity in those parts of the RF with the most direct access to the oromotor nuclei.

A specific rostrocaudal organization of PCRt has been recently proposed by Ter Horst et al. (1991) in which the rostral PCRt (rostral to $\mathrm{mVII}$ ) is hypothesized to control orofacial movement, whereas the caudal PCRt (caudal to mVII) controls metabolic homeostasis. Our results do not support this functional distinction of PCRt, because most of the FLI was located in the caudal PCRt. In fact, in the "rostral" PCRt at the level of mVII (level 6), the number of FLI neurons decreased substantially in all groups.

The lateral clustering of FLI neurons in IRt, together with the medial clustering in PCRt, formed a distinctive band of label consistently observed after quinine stimulation. In NST, quinineelicited FLI was distributed in the medial half of the rostral central subnucleus (Harrer and Travers, 1996). This continuous medial cluster of label from NST into the RF resembles a columnar organization that has been described in the goldfish vagal lobe. This structure is organized such that sensory layers project directly inward to motor layers, forming functional "columns" of neurons (Finger, 1988). In the rat, the NST seems to also project to the immediate subjacent RF (Becker, 1992; Halsell et al., 1996). The c-fos data, together with the NST efferent data, thus suggest that the NST-RF circuitry controlling rejection in rat may be functionally organized in a similar columnar fashion.

\section{REFERENCES}

Almeida A, Tjolsen A, Lima D, Coimbra A, Hole K (1996) The medullary dorsal reticular nucleus facilitates acute nociception in the rat. Brain Res Bull 39:7-15.

Amri M, Lamkadem M, Car A (1991) Effects of lingual nerve and chew- ing cortex stimulation upon activity of the swallowing neurons located in the region of the hypoglossal motor nucleus. Brain Res 548:149-155.

Anton F, Herdegen T, Peppel P, Leah JD (1991) c-FOS-like immunoreactivity in rat brainstem neurons following noxious chemical stimulation of the nasal mucosa. Neuroscience 41:629-641.

Barajon I, Gossard JP, Hultborn H (1992) Induction of fos expression by activity in the spinal rhythm generator for scratching. Brain Res 588:168-172.

Becker DC (1992) Efferent projections of electrophysiologically identified regions of the rostral nucleus of the solitary tract in the rat. Masters thesis, Ohio State University.

Beckman ME, Whitehead MC (1991) Intramedullary connections of the rostral nucleus of the solitary tract in the hamster. Brain Res 557:265-279.

Bellavance LL, Beitz AJ (1996) Altered c-fos expression in the parabrachial nucleus in a rodent model of CFA-induced peripheral inflammation. J Comp Neurol 366:431-447.

Bernard JF, Villanueva L, Carroue J, LeBars D (1990) Efferent projections from the subnucleus reticularis dorsalis (SRD): a Phaseolus vulgaris leucoagglutinin study in the rat. Neurosci Lett 116:257-262.

Berntson GG, Micco DJ (1976) Organization of brainstem behavioral systems. Brain Res Bull 1:471-483.

Borison HL, Wang SC (1949) Functional localization of central coordinating mechanism for emesis in cat. J Neurophysiol 12:305-313.

Brodal A (1957) The reticular formation of the brain stem: anatomical aspects and functional correlations. London: Oliver and Boyd.

Car A, Amri M (1987) Activity of neurons located in the region of the hypoglossal motor nucleus during swallowing in sheep. Exp Brain Res 69:175-182.

Carr PA, Huang A, Noga BR, Jordan LM (1995) Cytochemical characteristics of cat spinal neurons activated during fictive locomotion. Brain Res Bull 37:213-218.

Carstens E, Saxe I, Ralph R (1995) Brainstem neurons expressing c-Fos immunoreactivity following irritant chemical stimulation of the rat's tongue. Neuroscience 69:939-953.

Contreras RJ, Henderson R, Harrell HS (1996) Cardiovascular responses during taste-mediated licking in rats. Chem Senses 21:589.

DiNardo LA, Travers JB (1994) Hypoglossal neural activity during ingestion and rejection in the awake rat. J Neurophysiol 72:1181-1191.

Dobbins EG, Feldman JL (1995) Differential innervation of protruder and retractor muscles of the tongue in rat. J Comp Neurol 357:376-394.

Dragunow M, Faull R (1989) The use of c-fos as a metabolic marker in neuronal pathway tracing. J Neurosci Methods 29:261-265.

Emond MH, Weingarten HP (1995) Fos-like immunoreactivity in vagal and hypoglossal nuclei in different feeding states: a quantitative study. Physiol Behav 58:459-465.

Feldman JL, Ellenberger HH (1988) Central coordination of respiratory and cardiovascular control in mammals. Annu Rev Physiol 50:593-606.

Finger TE (1988) Sensorimotor mapping and oropharyngeal reflexes in goldfish, Carassius auratus. Brain Behav Evol 31:17-24.

Fraser KA, Raizada E, Davison JS (1995) Oral-pharyngeal-esophageal and gastric cues contribute to meal-induced c-fos expression. Am J Physiol 268:R223-R230.

Fukuda H, Koga T (1991) The Botzinger complex as the pattern generator for retching and vomiting in the dog. Neurosci Res 12:471-485.

Gieroba ZJ, Blessing WW (1994) Fos-containing neurons in medulla and pons after unilateral stimulation of the afferent abdominal vagus in conscious rabbits. Neuroscience 59:851-858.

Gieroba ZJ, Yu YH, Blessing WW (1994) Vasoconstriction induced by inhalation of irritant vapour is associated with appearance of Fos protein in $\mathrm{C} 1$ catecholamine neurons in rabbit medulla oblongata. Brain Res 636:157-161.

Grill HJ, Norgren R (1978a) The taste reactivity test. I. Mimetic responses to gustatory stimuli in neurologically normal rats. Brain Res 143:263-279.

Grill HJ, Norgren R (1978b) The taste reactivity test. II. Mimetic responses to gustatory stimuli in chronic thalamic and chronic decerebrate rats. Brain Res 143:281-297.

Guyenet PG (1990) Role of the ventral medulla oblongata in blood pressure regulation. In: Central regulation of autonomic functions (Loewy AD, Spyer KM, eds), pp 145-167. New York: Oxford University.

Halsell CB, Travers JB, Travers SP (1993) Gustatory and tactile stimulation of the posterior tongue activate overlapping but distinctive regions within the nucleus of the solitary tract. Brain Res 632:161-173. 
Halsell CB, Travers SP, Travers JB (1996) Ascending and descending projections from the rostral nucleus of the solitary tract originate from separate neuronal populations. Neuroscience 72:185-197.

Harrer MI, Travers SP (1996) Topographic organization of Fos-like immunoreactivity in the rostral nucleus of the solitary tract evoked by gustatory stimulation with sucrose and quinine. Brain Res 711:125-137.

Hathaway CB, Hu JW, Bereiter DA (1995) Distribution of Fos-like immunoreactivity in the caudal brainstem of the rat following noxious chemical stimulation of the temporomandibular joint. J Comp Neurol 356:444-456.

Herdegen T, Leah JD, Manisali A, Bravo R, Zimmermann M (1991) c-JUN-like immunoreactivity in the CNS of the adult rat: basal and transynaptically induced expression of an immediate-early gene. Neuroscience 41:643-654.

Hoffman GE, Le WW, Abbud R, Lee WS, Smith MS (1994) Use of Fos-related antigens (FRAs) as markers of neuronal activity: FRA changes in dopamine neurons during proestrus, pregnancy and lactation. Brain Res 654:207-215.

Holstege G, Kuypers HGJM, Dekker JJ (1977) The organization of the bulbar fibre connections to the trigeminal, facial and hypoglossal motor nuclei. Brain 100:265-286.

Houpt TA, Philopena JM, Wessel TC, Joh TH, Smith GP (1994) Increased c-fos expression in nucleus of the solitary tract correlated with conditioned taste aversion to sucrose in rats. Neurosci Lett 172:1-5.

Jasmin L, Gogas KR, Ahlgren SC, Levine JD, Basbaum AI (1994a) Walking evokes a distinctive pattern of Fos-like immunoreactivity in the caudal brainstem and spinal cord of the rat. Neuroscience 58:275-286.

Jasmin L, Wang H, Tarczy-Hornoch K, Levine JD, Basbaum AI (1994b) Differential effects of morphine on noxious stimulus-evoked Fos-like immunoreactivity in subpopulations of spinoparabrachial neurons. J Neurosci 14:7252-7260.

Jean A (1990) Brainstem control of swallowing: localization and organization of the central pattern generator for swallowing. In: Neurophysiology of the jaws and teeth (Taylor A, ed), pp 294-321. London: MacMillan.

Karimnamazi H, DiNardo LA, Travers JB (1994) Neurophysiological characterization of neuronal activity in the medullary reticular formation of the awake rat during ingestion. Soc Neurosci Abstr 20:817.

Karimnamazi H, Travers SP, Travers JB (1996) Descending projections from the gustatory responsive parabrachial nucleus to medullary reticular formation in the rat. Chem Senses 21:620-1.

Li YW, Dampney RAL (1994) Expression of Fos-like protein in brain following sustained hypertension and hypotension in conscious rabbits. Neuroscience 61:613-634.

Lowe AA (1981) The neural regulation of tongue movements. Prog Neurobiol 15:295-344.

Matsukawa K, Ninomiya I (1987) Changes in renal sympathetic nerve activity, heart rate and arterial blood pressure associated with eating in cats. J Physiol 390:229-242.

McEchron MD, McCabe PM, Green EJ, Hitchcock JM, Schneiderman N (1996) Immunohistochemical expression of the c-Fos protein in the spinal trigeminal nucleus following presentation of a corneal airpuff stimulus. Brain Res 710:112-120.

Mehler WR (1983) Observations on the connectivity of the parvicellular reticular formation with respect to a vomiting center. Brain Behav Evol 23:63-80.

Miller AD, Ruggiero DA (1994) Emetic reflex arc revealed by expression of the immediate-early gene c-fos in the cat. J Neurosci 14:871-888.

Moriyama Y (1987) Rhythmical jaw movements and lateral pontomedullary reticular neurons in rats. Comp Biochem Physiol 86A:7-14.

Nakamura Y, Katakura N (1995) Generation of masticatory rhythm in the brainstem. Neurosci Res 23:1-19.

Norgren R (1978) Projections from the nucleus of the solitary tract in the rat. Neuroscience 3:207-218.

Nozaki S, Iriki A, Nakamura Y (1993) Trigeminal premotor neurons in the bulbar parvocellular reticular formation participating in induction of rhythmical activity of trigeminal motoneurons by repetitive stimulation of the cerebral cortex in the guinea pig. J Neurophysiol 69:595-608.

Olson BR, Freilino M, Hoffman GE, Stricker EM, Sved AF, Verbalis JG (1993) C-Fos expression in rat brain and brainstem nuclei in response to treatments that alter food intake and gastric motility. Mol Cell Neurosci 4:93-106.

Onoda N (1992) Odor-induced fos-like immunoreactivity in the rat olfactory bulb. Neurosci Lett 137:157-160.
Parker LA (1994) Aversive taste reactivity: reactivity to quinine predicts aversive reactivity to lithium-paired sucrose solution. Pharmacol Biochem Behav 47:73-75.

Paxinos G, Watson C (1986) The rat brain in stereotaxic coordinates. San Diego: Academic.

Perreault MC, Rossignol S, Drew T (1994) Microstimulation of the medullary reticular formation during fictive locomotion. J Neurophysiol 71:229-245.

Rinaman L, Verbalis JG, Stricker EM, Hoffman GE (1993) Distribution and neurochemical phenotypes of caudal medullary neurons activated to express c-Fos following peripheral administration of cholecystokinin. J Comp Neurol 338:475-490.

Shammah-Lagnado SJ, Costa MSMO, Ricardo JA (1992) Afferent connections of the parvocellular reticular formation: a horseradish peroxidase study in the rat. Neuroscience 50:403-425.

Siegel JM, Tomaszewski KS (1983) Behavioral organization of reticular formation: studies in the unrestrained cat. I. Cells related to axial, limb, eye, and other movements. J Neurophysiol 50:696-716.

Soffe SR (1993) Two distinct rhythmic motor patterns are driven by common premotor and motor neurons in a simple vertebrate spinal cord. J Neurosci 13:4456-4469.

Steiner JE (1973) The gustofacial response: observation on normal and anencephalic newborn infants. In: Oral sensation and perception: development in the fetus (Bosma JF, ed), pp 254-278. Bethesda: Department of Health, Education, and Welfare.

Swank MW, Bernstein IL (1994) C-Fos induction in response to a conditioned stimulus after single trial taste aversion learning. Brain Res 636:202-208.

Swank MW, Schafe GE, Bernstein IL (1995) c-Fos induction in response to taste stimuli previously paired with amphetamine or $\mathrm{LiCl}$ during taste aversion learning. Brain Res 673:251-261.

Ter Horst GJ, Copray JCVM, Liem RSB, Van Willigen JD (1991) Projections from the rostral parvocellular reticular formation to pontine and medullary nuclei in the rat: involvement in autonomic regulation and orofacial motor control. Neuroscience 40:735-758.

Travers JB (1988) Efferent projections from the anterior nucleus of the solitary tract of the hamster. Brain Res. 457:1-11.

Travers JB, DiNardo LA (1992) Distribution of licking- and swallowingresponsive cells in the medullary reticular formation of the awake, freely moving rat. Soc Neurosci Abstr 18:1069.

Travers JB, Norgren R (1983) Afferent projections to the oral motor nuclei in the rat. J Comp Neurol 220:280-298.

Travers JB, Norgren R (1986) Electromyographic analysis of the ingestion and rejection of sapid stimuli in the rat. Behav Neurosci 100:544-555.

Travers JB, DiNardo LA, Karimnamazi H (1997) Motor and premotor mechanisms of licking. Neurosci Biobehav Rev, in press.

Travers JB, Montgomery N, Sheridan J (1995) Transneuronal labeling in hamster brainstem following lingual injections with herpes simplex virus-1. Neuroscience 68:1277-1293.

Travers SP (1993) Orosensory processing in neural systems of the nucleus of the solitary tract. In: Mechanisms of taste transduction (Simon SA, Roper SD, eds), pp 339-394. Boca Raton: CRC.

Travers SP, Norgren R (1995) Organization of orosensory responses in the nucleus of the solitary tract of the rat. J Neurophysiol 73:2144-2162.

Vertes RP, Martin GF, Waltzer R (1986) An autoradiographic analysis of ascending projections from the medullary reticular formation in the rat. Neuroscience 19:873-898.

Wallois F, Gros F, Masmoudi K, Larnicol N (1995) C-Fos-like immunoreactivity in the cat brainstem evoked by sneeze-inducing air puff stimulation of the nasal mucosa. Brain Res 687:143-154.

Wan W, Wetmore L, Sorensen CM, Greenberg AH, Nance DM (1994) Neural and biochemical mediators of endotoxin and stress-induced c-fos expression in the rat brain. Brain Res Bull 34:7-14.

Woods JW (1964) Behavior of chronic decerebrate rats. J Neurophysiol 27:635-644.

Yamamoto T, Shimura T, Sakai N, Ozaki N (1994) Representation of hedonics and quality of taste stimuli in the parabrachial nucleus of the rat. Physiol Behav 56:1197-1202.

Yousfi-Malki M, Puizillout JJ (1994) Induction of Fos-like protein in neurons of the medulla oblongata after electrical stimulation of the vagus nerve in anesthetized rabbit. Brain Res 635:317-322. 